\title{
Should we wait or not? The preferable option for patients with stage IV oral cancer in COVID-19 pandemic
}

\author{
Pankaj Chaturvedi, MS, FACS ${ }^{1}$ \\ ${ }^{1}$ Tata Memorial Centre and HBNI
}

April 28, 2020

\begin{abstract}
Background

The coronavirus infection is rapidly spreading putting a strain on healthcare services across the globe. Oral cancer patients are susceptible often immunosuppressed due to the disease and/or the treatment received.

Methods

We performed a simulationof the currently available data using a multi state and hazards model to provide an objective model for counseling and decision making for healthcare workers.

Results

Stage IV oral cancer patients that did not receive treatment had progression of disease and an increased mortality rate than patients that receive treatment but did not contract COVID-19. The patients that received treatment and got affected with COVID-19 had a far worse impact and higher mortality rate than all other groups.

Conclusion

Isolation and deferring treatment for stage IV oral cancer patients, so as to avoid hospital visits and contration of COVID-19, is an advisable strategy based on this model.
\end{abstract}

\section{Authors}

Atanu Bhattacharjee, PhD Section of Biostatistics Centre for Cancer Epidemiology Tata Memorial Centre and HBNI Email: atanustat@gmail.com

Vijay M Patil, MD Department of Medical Oncology Tata Memorial Centre and HBNI Email: vijaypgi@ gmail.com

Rajesh Dikshit, PhD Centre for Cancer Epidemiology Tata Memorial Centre and HBNI Email: dixr24@ gmail.com

Kumar Prabhash, MD Department of Medical Oncology Tata Memorial Centre and HBNI

Email: kprabhash1@gmail.com

Arjun Singh, MDS, MFDS (Glasgow) Department of Head Neck Oncology Tata Memorial Centre and HBNI Email: arjun193@gmail.com

Pankaj Chaturvedi*, MS, FACS Department of Head Neck Oncology Tata Memorial Centre and HBNI Email: chaturvedi.pankaj@gmail.com *Corresponding author

\section{Funding statement}

No funding was obtained from any sources. 


\section{Conflict of Interest statement}

None of the authors have any conflict of interest to declare.

\section{Authors contribution statement}

All authors have made a significant contribution to this article from concept to implementation and publication.

\section{Introduction}

As of the beginning of April, over a million people acrossthe globe have been tested positive for the novel severe acute respiratory syndrome corona virus 2 (SARS-CoV-2) infection ${ }^{1}$. Having begun in Wuhan,China, the epicentre of this pandemic has shifted to the USA over the past three months. Parallel large scale outbreaks have occurred in Italy and Spain with a majority of countries struggling to contain its spread.Cancer patients are believed to be one of the most vulnerable populations due to the immune compromised state caused by both the disease and its treatment. ${ }^{2}$

India has one of the largest incidence of oral cacner in the world. With the mounting evidence on COVID19,there are no systematic reports of cancer patients with COVID-19, let alone oral cancer. Patients with these cancers are more likely to succumb to COVID-19 than the cancer itself. It is believed that the SARS-COV-2 would accelerate cell death in a relatively short span of time, especially in patients receiving chemotherapeutic agents where the underlying immunity level is substantially low. Most healthcare administrators are deferring early stage diseases to be attended to after the situation subsides. The biggest brunt is being borne by the advanced stage oral cancer patietns, especially stage IV. Almost all of these tumors will progress to an unresectable stage by the time the pandemic is contained.Hence,planning of intervention and an adequate support strategy is required for the best service to be established. ${ }^{3}$

We present a simulation model using a multistate approach with transition-specific hazard functions that would predict the outcomes ofstage IV oral cancer patients thatreceive cancer directed treatment and get infected with SARS-CoV-2, and the same patients if they do not receive any cancer directed treatment and do not get infected with SARS-CoV-2during the pandemic.This model will provide a unique approach for setting suitable strategiestaking into account the current complex scenario of social distancing, human physiology and heterogeneity of the patients' disease status.

\section{Methods}

The conventional infectious disease model considers an exponentially rise in cases during the transition period. ${ }^{4}$ The ordinary differential equations are compatible to work with the exponential transition period model. The methodology of exponential time periodswasdoneusing the Gillespie algorithm. ${ }^{5}$ Since the time gaps are based on the patient's disease status, we divided theminto states based on the treatment received and infeced with SARS-CoV-2 andno treatment received and not infected with SARS-CoV-2, so that a hazard model could be applied. ${ }^{6}$ Model structures were applied through agent-based stochastic procedure. ${ }^{7}$

We assumed that patients with progressive disease and those receiving active treatment could not avoid a hospital vist and would continue rendering further treatment. Any of the above treatments would decline their immunity level from 0-100\%. Due to social distancing and stringentmobility criteria, we assumed that there were no follow-up visits and these patients would have a nil to minimal risk of contracting COVID-19. The states are expressed as $\mathrm{E}^{1}, E^{2}, E^{3}$ and $E^{4}$ dipicted in a directed acyclic graph(DAG). ${ }^{8}$ The corresponding time shift from one state to another is defined by time $T^{1}, T^{2}, T^{3}$ and $T^{4}$ respectively. (Figure 1)

A multistate model was used to specify the treatment initiation, disease progression, COVID-19 transmission and death. A cohort of stage IV oral cancer patients being treated or not were considered for analysis and their data was simulated. The transmission time was generated assuming that the transmission would occur during their hospital visit. The transmission probabilities and cumulative incidence were generated. The computation was performed using R software. The "mstate" and "muhaz" packages were used. 


\section{Multistate model}

Multi-state models are often used to describe the life history of an individual. It defines several possible events for a single individual or the dependence between several individuals. Events are considered when there is a transition between the states. This model is useful to represent an extremely flexible approach that can model almost any kind of longitudinal failure time data. ${ }^{9}$ Our model was formulated with states and transition steps. The DAG is formulated to describe the transition and time period for the transmission (Figure 2).

Patients that continue receiving treatment were defined as $E_{i}$, and there could be n number of patients theoretically. Another state that received no treatment is defined as $E_{j}$. Here, $E_{1}, E_{2}, E_{3}, E_{4}$ shows the transition from states $E_{i}$ to $E_{j}$ while $i<j$. We used the stochastic process defined as $\left(X_{(t)}\right), t \geq 0$ to explain the different states. The superset of states was defined as $\varepsilon=\left\{E_{1}, \ldots E_{n}\right\} . S_{i}=\inf \left\{t \geq 0 \mid X_{t}=E_{i}\right\}$.

We thenformulated the transition times of state $E_{j}$ from $E_{i}$ as $T_{j}=S_{j}-S_{\max \left\{k \mid k<j, S_{k}<\infty\right\}}$, assuming that $S_{0}=0$. The entire process was then defined by the transition time $T_{j}$ to state $E_{j}$. The hazard functions $h_{\mathrm{ij}}$ for the transition from $i$ to $j$ was defined as $\mathrm{T}_{\mathrm{ij}} \sim F_{\mathrm{ij}}=1-\exp \left\{-\int_{0}^{t} h_{\mathrm{ij}}(u) d u\right\}$ and $T_{j}=\min _{i \in\left\{1, \ldots, J-1 \mid T_{i}<\infty\right\}} T_{\mathrm{ij}}$. The cumulative distribution was presented as $\mathrm{F}_{\mathrm{ij}}$ for the transition from $E_{i}$ to $E_{j}$. Finally, all hazards were considered as constant X using the Markovian structure.

Hazards model

Assuming that the primary setups of the patients are presented with state $E_{1}$, further two states would be formulated as $\mathrm{E}_{2}$ and $\mathrm{E}_{3}$. The intermidiate and absorbing state would be defined as $E_{4}$.

The probability of transition from $E_{i}$ at time s to state $E_{j}$ at time $t$ is presented $\operatorname{asp}(s, t)=P\left[X=E_{j} \mid X_{s}=E_{i}\right]$ for $s \leq$ $t$.

If $i<j$ and $\neq j$, it may be formulated $\operatorname{asp} p_{\mathrm{ii}}(s, t)=\exp \left\{-\int_{s-S_{i}}^{t-S_{i}} h_{i 4}(u) d u\right\}$. Transition from $\mathrm{i}=4$ to $\mathrm{i}=4$ was not possible, but $i=1$ to $j=2,3,4$ were possible choices. Similarly, $i=2$ to $j=3,4$ were the other possibilities, i.e. $p_{i 4}(s, t)=1-\exp \left\{-\int_{s-S_{i}}^{t-S_{i}} h_{i 4}(u) \mathrm{du}\right\} \operatorname{and} p_{\text {ii }}(s, t)=0$.

Assuming that $S_{1}=0$, the transition probability can be obtained through integration

$$
\begin{gathered}
p_{11}(s, t)=\exp \left\{-\int_{s}^{t} h_{12}(u) d u-\int_{s}^{t} h_{13}(u) d u-\int_{s}^{t} h_{14}(u) \mathrm{d} u\right. \\
p_{12}(s, t)=\int_{s}^{t} p_{11}(s, u) h_{12}(u) p_{22}(u, t) \mathrm{du} \\
p_{13}(s, t)=\int_{s}^{t} p_{11}(s, u) h_{13}(u) p_{23}(u, t) \mathrm{du} \\
p_{14}(s, t)=1-p_{11}(s, t)-p_{12}(s, t)-p_{13}(s, t)
\end{gathered}
$$

This process was defined as $\mathrm{X}$ for state $E_{1}$ shifting from time $\mathrm{s}$ to $\mathrm{u}$. The states $E_{2}$ or $E_{3}$ will move from time $t$. The transition probability $p_{12}$ and $p_{13}$ can be obtained by calculating the integration over $u$.This integration can be obtained by simulating the transition time linked to the probabilities (Figure 3).

\section{Simulation of Hazards}

We simulated the hazard function to understand the magnitude of mortality. The transition specific hazard function was formulated with the assumption that hazard withmean $\mu=4$ will specify the constant function with $h(t)=\frac{1}{\mu}$ having parameter $\mu=4$. The time points $T_{\mathrm{ik}}$ by the hazard functions can be explained in the DAG (Figure 2). The minimum time period for shifting one state to another state is represented as prefixed k.It was possible to take a minimum of $\mathrm{k}$. If the transition time is $T_{j}$ and connecting state $E_{j}$. Therefore, 
we can simulate $\mathrm{X}$ from the initial state $T_{1 k}$ to calculate the first transition within a minimum period. The simulation then could be obtained by the corresponding state $E_{j}$. It was iterated until it became nil at the end of the simulation.

The hazard function was formulated as $h(t)$ with piecewise constant function $h_{\mathrm{pc}}(t)$. Using the msm package for simulation, the transition probabilities from the first state at time $t=0$ by the process $\mathrm{X}$ was calculated. ${ }^{10}$ We then simulated for N (Figure 4). Similarly, we used real data obtained from the website (https://ourworldindata.org/) and the data available on the coronavirus positive cases in India. This data is presented for the general population. The simulated portion for cancer patients' data is included for comparison. ${ }^{11,12}$ (Figure 4)

\section{Results}

Data simulated for cancer patients are plotted in Figure 3 and Figure 4. This model is prepared for a oncology hospital setup and simultaneously having the pressure to deal with COVID-19. The appearance of cancer cases is natural and the cumulative number is presented by the red line in Figure 3. This is a non-communicable disease that will report at its own pace, and the mortality rate without treatment will merge in Figure 3. But an alarming situation is present as the presence of COVID-19 is added. Probability of cancer patients getting infected by SARS-CoV-2for the next sixty days is plotted as a red line in Figure 4. Similarly, their probability of death is plotted as a black line in Figure 4.

Further, we separated them into two groups i.e. group A and group B(Figure 5). Group-A represents patients undergoing cancer directed treatment during the pandemic, and group B were those that were restricted dueto health serveicesnot rendering treatment. The 30 day mortality rate in our hosptial audit was $0.9 \%$ after completion of treatment. ${ }^{13}$ However, there is no data to suggest the probability of death when no treatment is rendered to stage-IV oral cancer patients. We accumulated the number of patients that join the pool as untreated(Group B). Even if we consider all these patients eventually die, the risk of death within the 60 days is lesser than those that contract SARS-CoV-2 and die. In absence of treatment the disease will progress and increase mortality, but does not exceed the mortality of those infected with SARS-CoV-2. The simulated presentation on group A and B demonstrated different scenarios. We should prefer to defer cancer treatement in these patients restricting the mortality to them.

The current patient load in our hospital is between 800-1000. Each day, 150 patients are registered on average. In the past 3 weeks, a total of 4150 should have been registered. The accumulated cancer mortality of partients not being treated (Group B) will be inclined linearly and we can expect that it can come close to 50. We assumed that Group B will see a cumulative increase of $10 \%$ in the rate of patients dying. In contrast, once infected by COVID-19, we expect to see a $50 \%$ cumulative increasein patients dying in group A. The soultion could revoles around treating the cancer patient efficiently with minimal clinic visits reducing their chance of infection.

\section{Discussion}

Countries across the world are implementing measures from national quarantines to school closures, to slow down the spread of the coronavirus. More than a third of humanity is under some form of restriction. ${ }^{14} \mathrm{~A}$ few reports have described cancer patients in quarantined cities struggling to obtain cancer directed services and essential medication. ${ }^{15}$ Over time, this will exacerbate due to interruptions in scheduled surgeries, chemotherapy and radiotherapy in order to prioritize hospital space and care for the seriously ill COVID-19 patients.

Among the cancer patients who develop COVID-19, treatment of the cancer will be delayed in order to prioritize treatment for the infection. Many physicians and surgeons around the world are being forced to take this tough call on an individual patient basis. With these limited resources and capacity it is important to understand the implications it has on cancer patients with and without COVID-19. Given the acute time frame of COVID-19 infection and lack of any retrograde data, a prospective study of scale cannot be accomplished. The challenge comes about when we need to triage cancer care during this pandemic. In 
our analysis we assumed that patients treated for cancer will have a lesser risk of contracting the virus at the hospital due to the measures taken by the governments. At the same time their chances of disease inflammation and death are relatively high due to the cancer or the treatment received.

The simulated work presented here is to visualize the impact of COVID-19 in the worst case scenario so as to support policy makersmake the hard decisions. Nonetheless, decisions on patients care is required to be taken as per the case, rather than abide by blanket guidelines. Due to the limitation of adequate real world data and follow up on COVID-19, we cannot provide personalized recommendations regarding cancer care. The increased mortality of patients seen in this model should caution oncologists. The risk-benefit ratio should be discussed with patients before administering any definitive treatment. We propose that forceful steps are required to reduce the frequency of hospital visits for patients having cancer during this time.Proper isolation techniques are required to mitigate the risk of transmission. Risk factors like disease severity, recent chemotherapy received can increase the chance of illness and mortality due to COVID-19.It is also required to take steps to eliminate cross-infection between the patients and healthcare workers.

With the situation gradually unfolding, there is not much we can do as health care providers but to adapt as best we can. In case of suspicion, appropriate isolation techniques need to be instated. Among cancer patients, receiving recent chemotherapy does increase the risk of severe illness. This model helps us better understand the survival trajectory of oral cancer patients that might be affected by COVID-19.

\section{References}

1. Coronavirus disease 2019 (covid-19) situation report^a_A_T43. https://www.who.int/docs/default source/coronaviruse/situation-reports/20200303-sitrep-43-covid-19.pdf?sfvrsn=2c21c09c2:00.

2. C. P. E. R. E. Novel et al., \The epidemiological characteristics of an outbreak of 2019 novel coronavirus diseases (covid-19) in china," Zhonghualiuxingbingxue za zhi= Zhonghualiuxingbingxuezazhi, vol. 41, no. 2, p. $145,2020$.

3. Y. Yamanaka, K. Uchida, M. Akashi, Y. Watanabe, A. Yaguchi, S. Shimamoto, S. Shimoda, H. Yamada, M. Yamashita, and H. Kimura, \Mathematical modeling of septic shock based on clinical data,"Theoretical Biology and Medical Modelling, vol. 16, no. 1, p. 5,2019.

4. R. M. Anderson, B. Anderson, and R. M. May, Infectious diseases of humans: dynamics and control. Oxford university press, 1992.

5. D. T. Gillespie, Exact stochastic simulation of coupled chemical reactions," The journal of physical chemistry, vol. 81 , no. 25, pp. 2340-2361, 1977.

6. A. L. Lloyd, Realistic distributions of infectious periods in epidemic models: changing patterns of persistence and dynamics," Theoretical population biology, vol. 60, no. 1, pp. 59\{71, 2001.

7. J. Estill, C. Aubriere, M. Egger, L. Johnson, R. Wood, D. Garone, T. Gsponer, G. Wandeler, A. Boulle, M.-A. Davies et al., Viral load monitoring of antiretroviral therapy, cohort viral load and hiv transmission in southern africa: a mathematical modelling analysis," AIDS (London, England), vol. 26, no. 11, p. 1403, 2012.

8. J. Pearl et al., Causal inference in statistics: An overview," Statistics surveys, vol. 3, pp. 96-46, 2009.

9. P. Hougaard, Multi-state models: a review," Lifetime data analysis,vol. 5, no. 3, pp. 239-264, 1999.

10. C. H. Jackson et al., \Multi-state models for panel data: the msm package for r," Journal of statistical software, vol. 38, no. 8, pp. 1-29, 2011.

11. Our World in Data [Internet]. [cited 2020 Apr 10]. Available from: https://ourworldindata.org/

12. \#IndiaFightsCorona COVID-19 [Internet]. MyGov.in. 2020 [cited 2020 Apr 10]. Available from: https://mygov.in/covid-19/

13. Nair Deepa et al.,Outcomes of surgically treated oral cancer patients at a tertiary cancer center in India.Indian Journal of cancer, vol. 54, no. 4, pp. 616-620, 2017.

14. CDC. Coronavirus Disease 2019 (COVID-19) in the U.S. [Internet]. Centers for Disease Control and Prevention. 2020 [cited 2020 Mar 31]. Available from: https://www.cdc.gov/coronavirus/2019ncov/cases-updates/cases-in-us.html

15. Al-Shamsi HO, Alhazzani W, Alhuraiji A, Coomes EA, Chemaly RF, Almuhanna M, et al. A Practical 
Approach to the Management of Cancer Patients During the Novel Coronavirus Disease 2019 (COVID19) Pandemic: An International Collaborative Group. The Oncologist. 2020 Apr 3;

Figure legend:

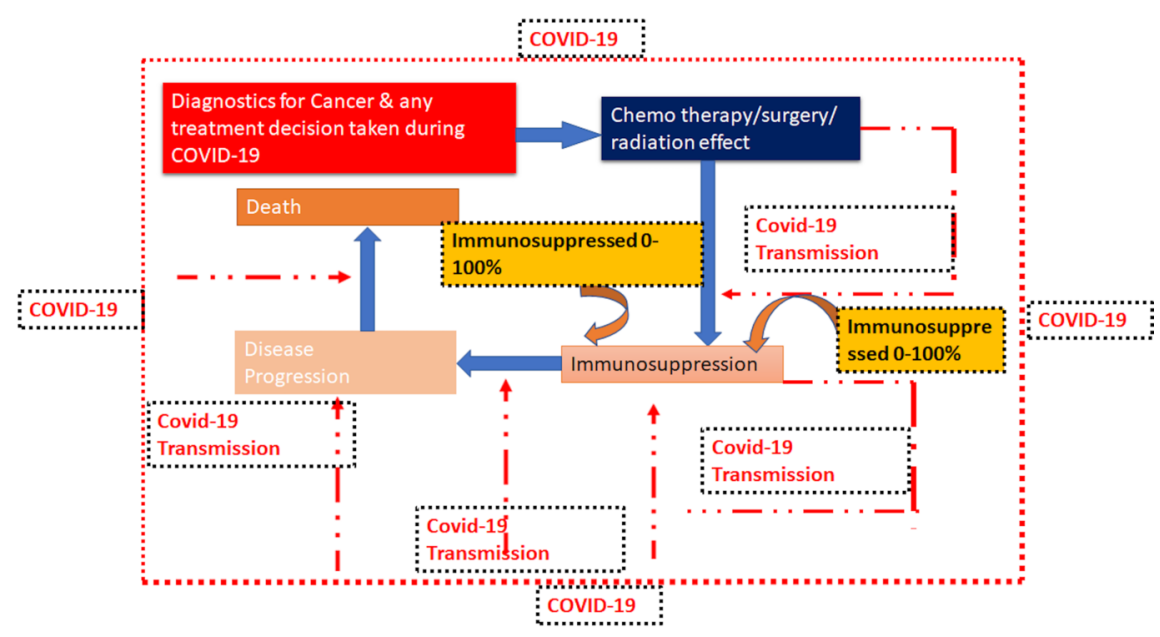

Figure 1: This is a caption

Figure 1: Overview of COVID-19 transmission among stage IV oral cancer patients

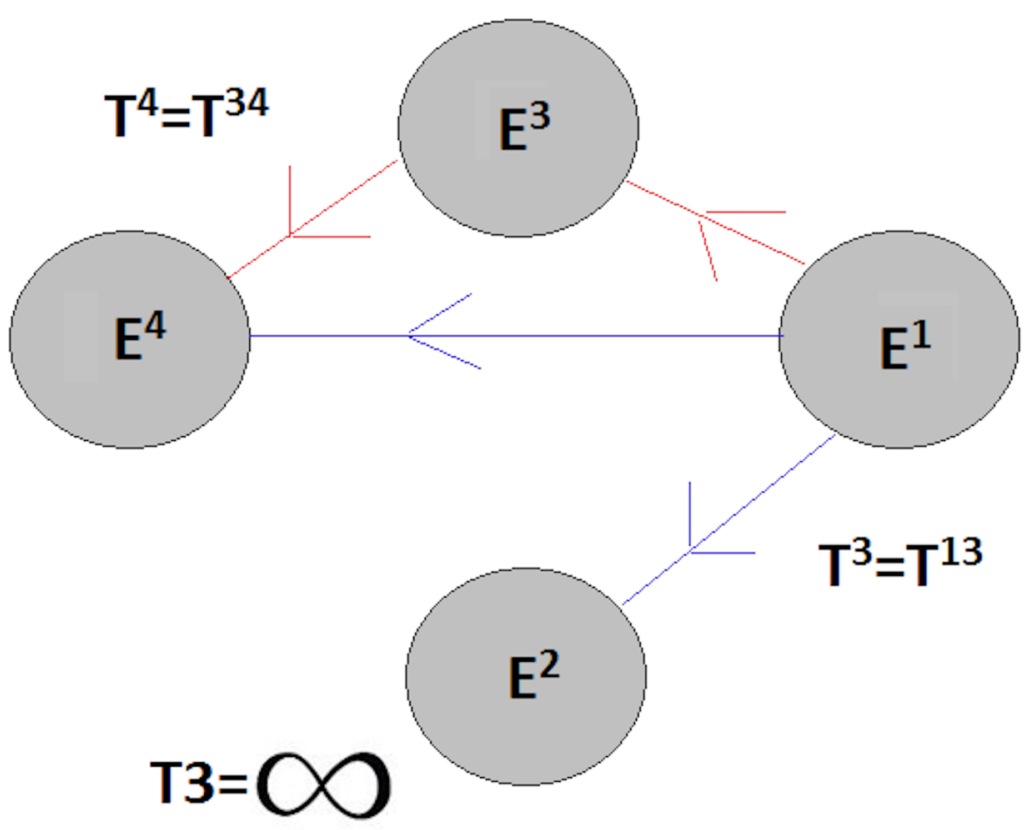

Figure 2: This is a caption 
Figure 2: Multistate model transmission of COVID-19 among stage IV oral cancer patients

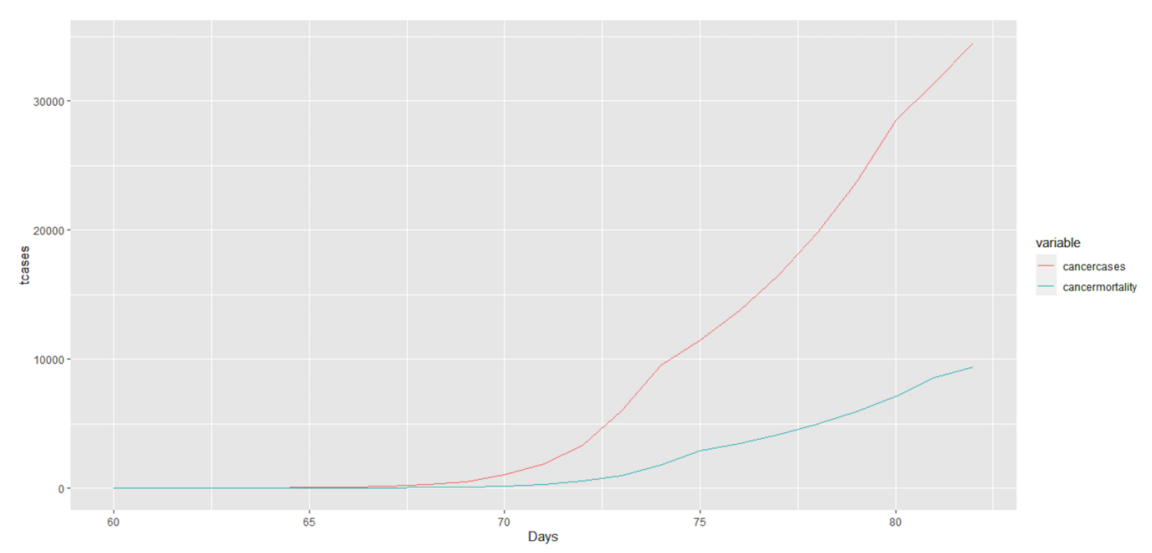

Figure 3: This is a caption

Figure 3: Outcomes of patients with cancer and cancer mortality

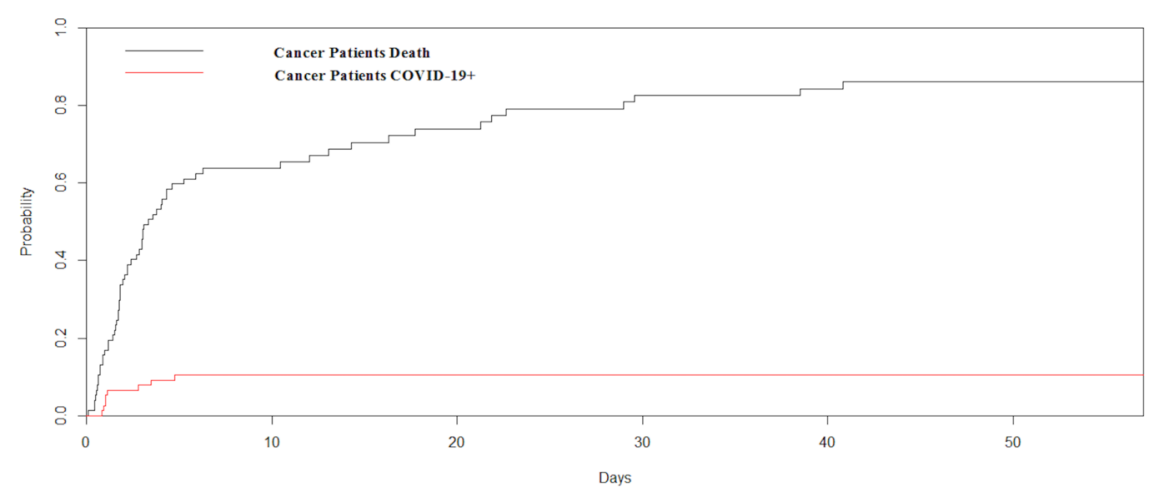

Figure 4: This is a caption

Figure 4: Comparison of patients affected by COVID-19 and cancer patients mortality comparison 


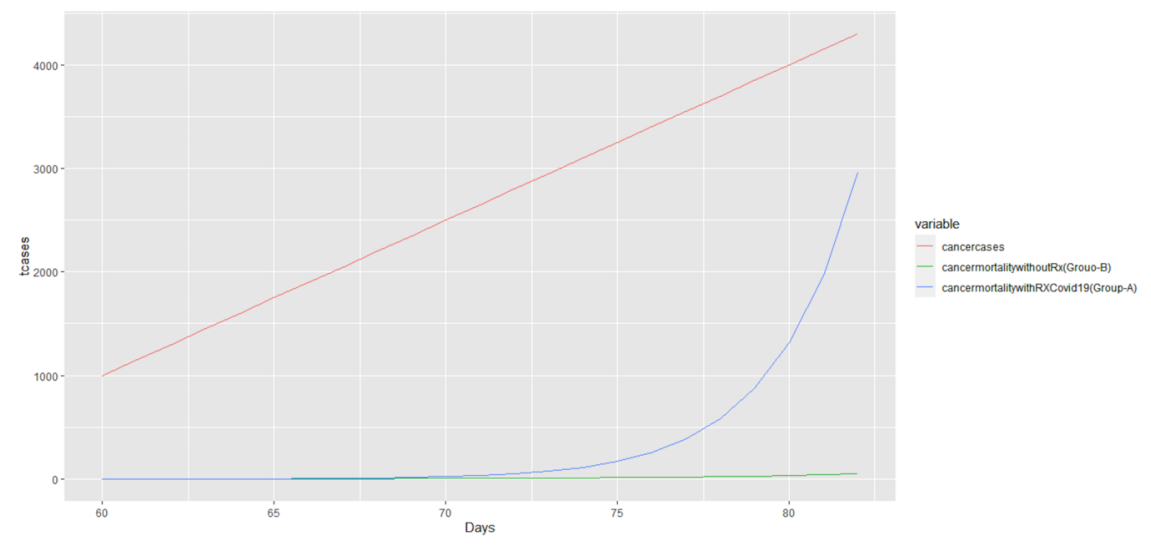

Figure 5: This is a caption

Figure 5: Comparision of patients receiving treatment and being infected by COVID-19 and patients not receiving and cancer directed treatment 